\title{
A COMPUTATIONAL STUDY OF THE DIFFUSE NEIGHBOURHOODS IN BIOLOGICAL AND ARTIFICIAL NEURAL NETWORKS
}

\author{
P. Fernández López, C. P. Suárez Araujo \\ Institute for Cybernetics, University of Las Palmas de Gran Canaria, Spain \\ pfernandez@dis.ulpgc.es,cpsuarez@dis.ulpgc.es \\ P. García Báez \\ Dept. of Statistics, Operations Research and Computation, University of La Laguna, Spain \\ pgarcia@ull.es
}

Keywords: Nitric oxide, Artificial neural networks, Cellular signalling, Volume transmission, Diffuse neighbourhood.

\begin{abstract}
This paper presents a computational study on a fundamental aspect concerning with the dynamic of nitric oxide (NO) both in the biological and artificial neural networks, the Diffuse Neighbourhood (DNB). We apply the compartmental model of NO diffusion as formal tool, using a computational neuroscience point of view. The main objective is the analysis of DNB by the observation of the AI-NOD and CDNB variables, defined in this work. We present a study of influences and dependences with respect to associated features to the NO synthesis-diffusion process, and to the environment where it spreads (non-isotropy and nonhomogeneity). It is structured into three sets of experiences which cover the quoted aspects: influence of the NO synthesis process, isolated and multiple processes, influence of distance to the element where NO is synthesized, influence of features of the diffusion environment. The developments have been performed in mono and bi-dimensional environments, with endothelial cell features. The importance of this study is providing the needed formalism to quantify the information representation capacity that a type of NO diffusion-based signalling presents and their implications in many other underlying neural mechanisms as neural recruitment, synchronization of computations between neurons and in the brain activity in general.
\end{abstract}

\section{INTRODUCTION}

The understanding of brain structure and function and its computational style is one of the biggest challenges both in Neuroscience and Neural Computation. To reach this aim it is essential to know underlying mechanisms of the brain activity.

The activity of the brain has as principal responsible the $\mathrm{BNN}$, the cellular communication and learning. Neural recruitment, or synchronization of computations between neurons, the existence of an information indexing schema at the Biological Neural Network (BNN), or the LTP expression, are aspects that can depend in a direct way on an underlying neural signalling schema. We understand that such aspects will be able to have a considerable role in the information representation capacity, and so, in the BNN and ANN computation potential.
Among all set of cellular signals that affect globally the brain activity, the volume transmission (VT) is located. Its underlying mechanism is the diffusion of neuroactive substances and diffusible signals, like Nitric Oxide (NO). NO is one of the liposoluble molecules generated by cells from the own tissue which allow a volumetric transmission. A key property of NO is its extreme diffusibility in both aqueous and lipid environments, which allows a fast three-dimensional spread of the signal irrespective of the presence of membranes (Suárez Araujo, 2000). Because of this, it freely diffuses through membranes affecting all neighbouring cells (Hawkins, et al., 1993), (Schuman, et al., 1994), (Zhuo, et al., 1998) and (Garthwaite, et al., 1995).

The presence of a molecule in the brain such as $\mathrm{NO}$, opens new perspectives in the study of the brain functioning. NO can help as an element of control for several systems. It can act as a retrograde neurotransmitter; it can be involved in learning and 
memory, and in the LTP process. It is capable to produce hybrid neuromodulation, diffusive hybrid neuromodulation (DHN) (Suárez Araujo, 2000). NO has also opened a new dimension in our concept of neural communication, overlaying the classical synaptic neurotransmission, where information is passed between neuronal elements at discrete loci (synapses).

An intrinsic feature of the NO diffusion is the formation of not-wired neighbourhoods, diffuse neighbourhoods (DNB), which supports the emerging of complex structures. The formation of these structures has been a subject studied by other authors (Krekelberg, 1996, 1997) and (Krekelberg and Taylor, 1998). These studies have been specific studies concerning with the cortical map formation and their relationship with neighbourhoods in the Kohonen SOM. They have not considered the DNB as a possible underlying communication schema in BNN and ANN. Our studies consider this capacity.

Our work introduces and studies fundamental concepts in the formation of DNB, product of the dynamics of NO diffusion. We understand DNB as an auto-contained element in the behavior of NO and the study of its dynamics as essential, independent from the learning model or the neural architecture where it is embodied in. This will provide us a better understanding of the computational skills that NO has; computational skills based on the neural recruitment mechanisms, emergence of complex structures and increase of information capacity and processing. This justifies the need of a generalist and in-depth development like the one our work presents. We provide concepts located in the Theoretical Framework of the study of NO (Suárez Araujo, 2000). It will be able to manage studies of causal aspects of the NO dynamics, and comparative studies with concepts and classical architectures.

In this paper, we focus our effort on the analysis of DNB dynamics and its possible influence in mechanisms and processes at the neural circuit and/or higher level. An important aim is to infer from the analysis a possible implication of VT in the increase of the information representation capacity in both BNN and ANN, in their architectures and in the functional complexity of its main computation element, the neuron.

We present a computational analysis of DNB, based on our compartmental model of diffusion of NO (Suárez Araujo, et al., 2006). This work requires of a set of own concepts of the diffusive phenomena, which we have defined and that are: Directionality of NO dynamics (DNO), Average Influence (AI),
Diffusion Centre of the DNB (CDN) and DNB Limit (DNBL).

The importance of this study is providing the needed formalism to quantify the information representation capacity that a type of NO diffusionbased signalling can present.

\section{METHOD}

Diffusion is the main axis in the study of the NO dynamics (Wood and Garthwaite, 1994), as well as the main responsible of the NO influence to different brain zones from a functional and structural point of view. This influence is materialized, essentially, by means of the Diffuse Neighbourhoods (DNB). This concept allows to analyze how NO influences move by means of diffusion as well as what is their dependence with non-isotropy and nonhomogeneity. The establishment and analysis of DNB, which we will perform using the compartmental model of NO diffusion, precise to formalize intrinsic aspects to the diffusion phenomena and to the NO dynamics. On one hand, we have the directionality measure of the NO dynamics, which provokes different spatial-temporal influences in the diffusion environment. This takes us towards the concept of Average NO Influence, key variable in the DNB definition. On the other hand, its dynamic, adaptive and no local character justifies the need of variables which formalize that dynamism and its effect as diffusion centre of the DNB and DNB limit.

\subsection{Compartmental Model of NO Diffusion and Concepts of Diffusion}

The compartmental model of NO diffusion (Suárez Araujo, et al., 2006) is a discrete computational model that allows us to study the dynamic of NO, generation, diffusion, self-regulation and recombination, in biological and artificial environments. Its main feature is its simplicity, it can be considered as a general formal tool with biological plausibility. It gathers real features of the diffusion environment such as the no homogeneity and the no-isotropy and possible morphology of the NO synthesis.

It represents an important tool for designing and interpreting biological experiments on NO behaviour and its effect on brain structure and function.

The model is based in compartmental systems (Anderson, 1983) and it is defined by a system of 
first order differential equations, like eq. (1), where we can consider specific cyclic contour conditions.

$$
\frac{d C_{i}}{d t}=D_{i, i-1}\left(C_{i-1}-C_{i}\right)+D_{i, i+1}\left(C_{i+1}-C_{i}\right)-\lambda_{i} C_{i}+F_{i}
$$

Where $D_{i, i-1}$ and $D_{i, i+1}$ are the coefficients of diffusion between the compartments $i$ and $i-1$ and between $i$ and $i+1$, respectively. $\lambda_{i}$ is the selfregulation parameter of NO. It is being considered, for this case, a self-regulation of NO dynamics proportional to the quantity of concentration, and $F_{i}$ is the function of generation of NO.

The computational analysis of DNB, using this model requires of a set of own concepts of the diffusion phenomena, which will be defined and that are: Directionality of NO dynamics (DNO), Average Influence (AI), Diffusion Centre of the DNB (CDN) and DNB Limit (DNBL).

\section{NO Directionality, (NOD)}

NOD allows to assign an $i$ state to every compartment, associated to the movement that NO has along each dimension. Basing on this, it can be measured the permanence time of compartment $i$, in such state, and to calculate the Average NO Influence that a source $i$ compartment can be causing on a destination compartment $j$.

NOD is formally defined for an $i$ compartment by equation 2. Its value is function of the NO concentration dynamics associated to the adjacent compartments, according to the propagation schema defined for each dimension.

$$
\begin{aligned}
\frac{d G_{i}}{d t} & =D_{i+1, i+2} C_{i+2} \\
& -\left(D_{i+1, i}+D_{i+1, i+2}+\lambda_{i+1}\right) C_{i+1} \\
& +\left(D_{i+1, i}-D_{i-1, i}\right) C_{i} \\
& +\left(D_{i-1, i}+D_{i-1, i-2}+\lambda_{i-1}\right) C_{i-1} \\
& -D_{i-1, i-2} C_{i-2} \\
& +F_{i+1} \\
& -F_{i-1}
\end{aligned}
$$

Basing on the NOD value in each dimension, an $i$ compartment can be located on 3, 9 or 27 states, for environments with one, two or three dimensions, respectively, Table 1.

Basing on time proportion, over the total time of the diffusion process in which compartments are located in one state, it can be calculated the Average Influence that an $i$ compartment is performing into another $j$ compartment.
Table 1: Possible states of a compartment in monodimensional environments and its NOD values.

\begin{tabular}{|c|c|}
\hline State & $\mathrm{G}_{\mathrm{i}}$ Condition \\
\hline$\leftarrow$ & $\mathrm{G}_{\mathrm{i}}<0$ \\
\hline$\rightarrow$ & $\mathrm{G}_{\mathrm{i}}>0$ \\
\hline$\leftrightarrow$ & $\mathrm{G}_{\mathrm{i}}=0$ \\
\hline
\end{tabular}

\section{Average Influence (AI)}

Average Influence between compartments by the NO dynamics (AI) is a magnitude that quantifies the influence $k$ compartment is performing into $r$ compartment.

$$
I(k, r, t)=\max \left(\prod_{i \in T(k, p, r, T(p, q, r, T(\ldots)))} \psi\left(i, E_{T}, t\right)\right)
$$

Where $i$ is every compartment that is located on $T(k, p, r, T(p, q, r, T(\ldots)))$, path, definition-recursive, which goes from $k$ compartment to $r$ compartment, passing by intermediate compartment $p$ and using path $T(p, q, r, T(\ldots))$. The function $0<\psi\left(i, E_{T}, t\right)<1$ defines the time proportion, over the total process time $t$, that $i$ compartment is located on the $E_{T}$ state in favor of the direction followed that path $T$ defines. This magnitude is computed for a determined instant, being able to vary throughout time.

\section{Diffuse Neighbourhood (DNB)}

Diffuse Neighbourhood (DNB) of an $i$ compartment, (4), is constituted by a set of compartments which fulfill certain criteria with relation to the AI $i$ compartment is performing in them.

$$
V_{i}(t)=\{j: Q(I(i, j, t))\}
$$

Where $Q(I(i, j, t))$ is the $Q$ criteria over the AI that $i$ compartment performs in the $j$ one, and $j$ represents every compartment that fulfills $Q . Q$ can be the exceed of a threshold value $\theta$ by the AI. This way, $j \in V_{i}(t) \Leftrightarrow Q(I(i, j, t))$ is fulfilled, where $Q$ is defined according to the logical expression $I(i, j, t)>$ $\theta$. Another criterion could be that AI is located between two values, $\theta_{1}$ y $\theta_{2}$. So $Q$ criterion would be defined by the logical expression $\theta_{1}<I(i, j, t)<\theta_{2}$. The way in which $Q$ criterion is defined causes different types of neighbourhoods, and the DNB of a compartment can change throughout time. This way, the DNB is dynamic and adaptive that generates complex structures. These aspects of the DNB can be featured by tracing the diffusion centres of every instance of the DNB throughout time. This diffusion centre of the DNB (CDNB) determines a position, which corresponds to the averaged position of all the 
influences that compartments belonging to the neighbourhood perform between them

$$
\mathbf{C}\left(V_{i}, t\right)=\sum_{j \in V_{i}} I(i, j, t) \mathbf{r}_{j} / \sum_{j \in V_{i}} I(i, j, t)
$$

Where $\mathbf{r}_{\mathbf{j}}$ corresponds to the position $j$ compartment has in the diffusion media.

\section{Diffuse Neighbourhood Limit, (DNBL)}

We define Diffuse Neighbourhood Limit (DNBL) of an $i$ compartment as the $\max \left(\left|\mathbf{r}_{j}-\mathbf{r}_{i}\right|\right)$, where $\mathbf{r}_{i}$ is the position vector of the $i$ compartment, $\mathbf{r}_{j}$ is the position vector associated to the $j$ compartment, and such compartment corresponds to any of the compartments belonging to the DNB of the $i$ compartment $i, j \in V_{i}(t)$.

\section{RESULTS AND DISCUSSION}

We have performed a computational study of NO dynamics, using the compartmental model of $\mathrm{NO}$ diffusion by the observation of the AI-NOD and CDNB variables.

We have focused our efforts in the analysis of one of the great potentialities of $\mathrm{NO}$ as diffusive signalling, the emergency of DNB and so, of complex structures. We have established influences and dependences with respect to associated features to the NO synthesis-diffusion process, and to the environment where it spreads (non-isotropy and nohomogeneity) (Syková, 2001). We have structured the study into three sets of experiences which cover the quoted aspects: influence of the NO synthesis process, isolated and multiple processes, influence of distance to the element where NO is synthesized, influence of features of the diffusion environment, isotropy and homogeneity, presenting our obtained results in this section.

The first two studies have been performed in a mono-dimensional environment, figure 1, with endothelial cell features. The values of the diffusion and auto-regulation constants are, respectively, $D=$ 3,3.103 $\mathrm{\mu m}^{2} \mathrm{~s}^{-1}$ and $\lambda=1,3863 \mathrm{~s}^{-1}$ and average life $\mathrm{t}_{1 / 2}=0.5 \mathrm{~s}$. (Malinski, et al., 1993)

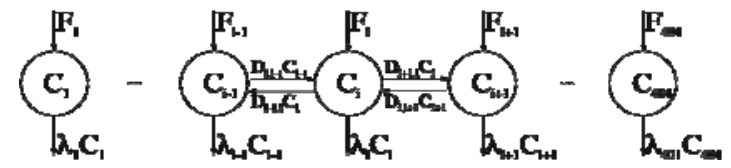

Figure 1: Environment of 401 compartments. The synthesis processes are in the compartment 201 for synthesis alone, and at 101, 201 and 301 for multiple syntheses.

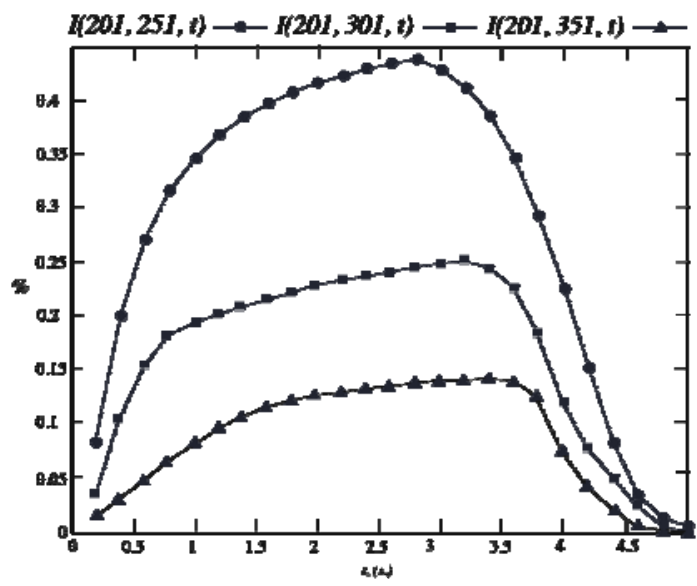

Figure 2: I(201, 251, t), I(201, 301, t), I(201, 351, t) AI Profiles that compartment 201 produces into compartments 251, 301, 351.

Figure 2 shows the AI which exerts the compartment 201 into compartments placed at different distances from it. It is observed the AI dependence with the distance to the NO-generator compartment and time. The synthesis process at compartment 201 starts at $t=0 \mathrm{~s}$., takes $0.2 \mathrm{~s}$. and presents trapezoidal morphology (Suárez, et al., 2006). We have observed that $I(201,251, t)$ gets its maximum, $I_{\max }(201,251, t) \cong 45 \%$, at $t \cong 2.75 \mathrm{~s}$. Initially, compartments produce influence into other compartments, in a fast way, the more the less distance. This is fundamental to propitiate neural recruitment processes, computations synchronizations, adaptations to changes of environment. Thus, when synthesis is over, there already exists an AI greater than $15 \%$ of $I_{\max }(201$, $251, t)$. After reaching its maximum, $I(201,251, t)$ decays at a faster speed, getting at $t=5 \mathrm{~s}$., negligible values. $I(201,301, t)$ takes a $25 \%$ more than $I(201$, $251, t)$ in reaching the maximum, and $I(201,351, t)$ a $30 \%$ more, being the max influences much lower in these compartments. When increasing the distance in $50 \mu \mathrm{m}$, the max AI value falls in a $50 \%$, and when increasing it in $50 \mu \mathrm{m}$. more, $I_{\max }$ decreases a $75 \%$, Figure 1. This analysis takes us to another concept as "the importance of neighbourhood" is threshold value of $I$ which compartments have to reach to consider them as belonging to a DNB. Thus, DNB are dynamic and adaptive, being formed gradually in time by means of the incorporation of compartments and, at the same time, modifying their order at the neighbourhood. Importance will be an indicator of the generated DNB stability; the more stable the DNB, the lower its importance. This changing character of DNB handles working in non-stationary environments, real environments. It can help the 
formation of cortical maps (Krekelberg, 1996) and (Krekelberg and Taylor, 1998), hybrid complex structures, DNB + wired neighbourhoods, as well as the incorporation of volume learning into BNN and ANN.

All these computation and/or information representation potentialities will be more justified with the emergence of complexity in DNB when we increase complexity in NO generation processes.

We have studied the dynamics in an environment where there coexist several simultaneous synthesis processes. We analyzed the AI in compartment 251, when synthesis process exists in two symmetrical positions to 251 at both sides. We have observed a dynamics where compartments with minimum influence exist. $I(201,251, t)$ as well as $I(301,251$, t) are lower than 3,5\%, Figure 3. Thus, a value of importance higher than this $3,5 \%$ implies this compartment does not belong to both neighbourhoods, even though it is at short distance from the NO generation. It can be understood that compartment 251 plays an isolator role of the several complex structures that are formed. This feature can indicate the existence of isolation zones in the biological level, which can cause computational segmentation and information indexing.

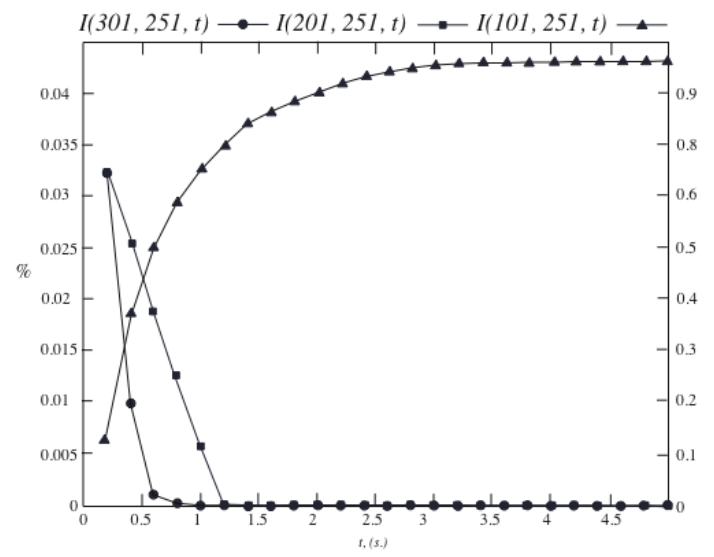

Figure 3: I(201, 251, t), I(301, 251, t), I(101, 251, t) AI Profiles that compartments 201 and 301 exert into compartment 251, and compartment 101 into compartment 251, when there exist two, and three, simultaneous synthesis processes, respectively.

It has been performed the study of the IM-NOD profiles throughout time, when 3 simultaneous NO synthesis processes exist, separated by $100 \mu m$. one another (compartments 101, 201 and 301). Analyzing the IM-MOD compartment 101 exerts into compartment 251 , it is observed the additive character that multiple and aligned generation causes, Figure 3. To thwart this effect, it becomes necessary the variability of the average NO lifetime in the neural tissue. This is one of the biological phenomena which can justify the environment nonhomogeneity.

Finally, it is shown the dependence that DNB dynamics and CDNB have to environment nonisotropy and non-homogeneity. We have worked in a bi-dimensional environment with very low values of the diffusion constant in two zones, which makes the NO dynamics to be almost null. The diffusion

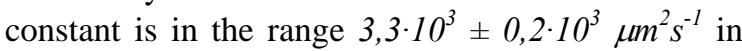
the rest of the environment. There are induced two NO synthesis processes in two compartments $i, k$. It is observed a formation of non-symmetrical and non-local $\mathrm{DNB}_{\mathrm{i}}$ and $\mathrm{DNB}_{\mathrm{k}}$ at times $t=0,6 \mathrm{~s}$. and $t=$ 1,3 s., Figures $4 \mathrm{a}$ and $4 \mathrm{~b}$. Thus it is noticed a changing trajectory in CDNB and its possible dependence in the way DNB does. For $t=0,6$ s., in both neighbourhoods, CDNB matches with the compartment where synthesis was caused; however for $t=1,3 \mathrm{~s}$. in $\mathrm{V}_{\mathrm{i}}$ the position of CDNB has changed, moving in agreement to that neighbourhood shape.

\section{CONCLUSIONS}

We present a work developed from a computational neuroscience point of view which provides a step forward in the understanding of the VT and their implications in the biological and artificial neural networks.

We have performed a computational analysis of one of the great potentialities of NO as diffusive signalling, the DNB. We have used the compartmental model of NO diffusion, showing its high capacity to study the dynamics of NO.

We have proposed and defined concepts associated to the diffusion phenomena which present significant capabilities to characterize the NO dynamics. These concepts are Directionality of NO dynamics, Average Influence, Importance of the Neighborhood, Diffusion Centre of the DNB and DNB Limit.

We have established that the generation and dynamical behavior of the DNB depend on associated characteristics to the NO synthesisdiffusion processes, and to the environment where it spreads (non-isotropy and non-homogeneity). The complexity in DNB emerges when the complexity in NO generation processes is increased. In this paper, it is also showed the existence of isolation zones in the biological level, which can cause computational 


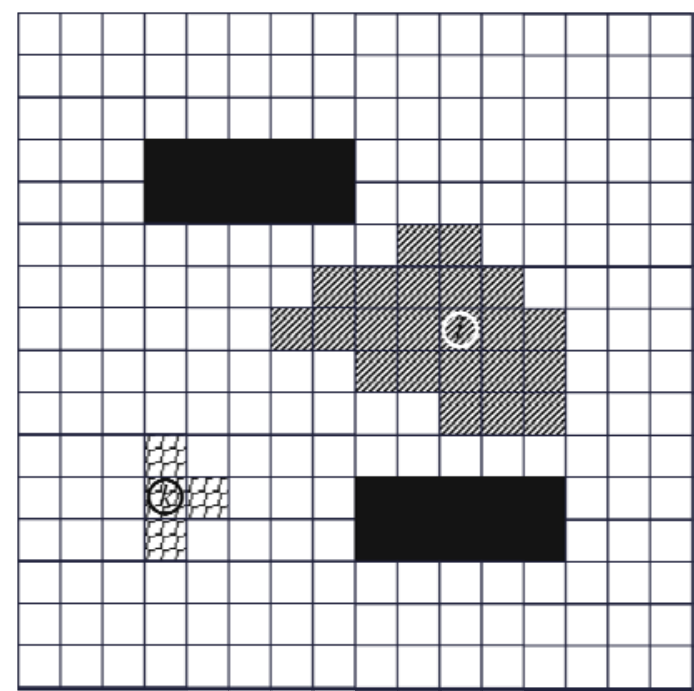

a)

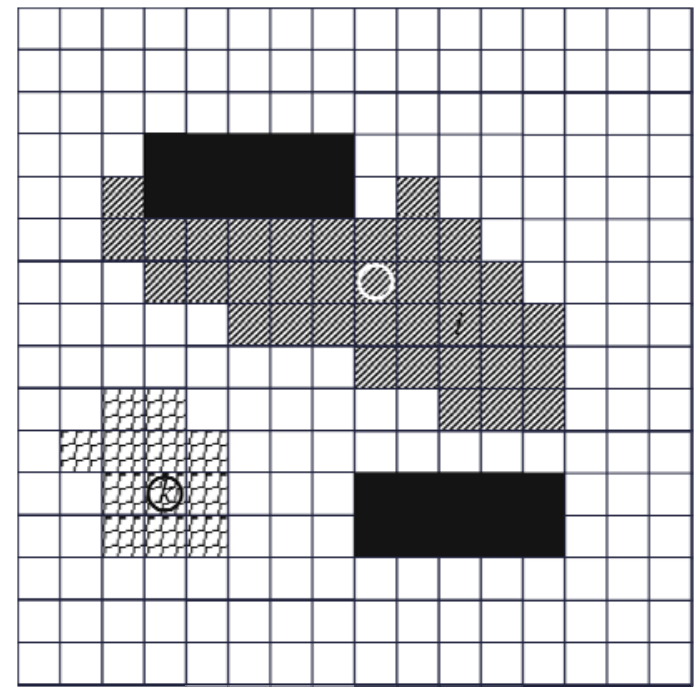

b)

Figure 4: Snapshot of the formation of two neighbourhoods, $\mathrm{DNB}_{\mathrm{i}}, \mathrm{DNB}_{\mathrm{k}}$ and position of $\mathrm{CDNB}$ (indicated by a circle) in different times. Non-isotropic and non-homogeneity environment of 16x16 compartaments. Black zone corresponds to null NO dynamics. a) $\mathrm{t}=0,6 \mathrm{s.}, \mathrm{b}) \mathrm{t}=1,3 \mathrm{~s}$.

segmentation and information indexing, and the possibility to generate non local and non symmetric DNB. With this study, it is possible to explain some important environment features like the nonhomogeneity. Finally, all these results allow us to detect the implications of VT, by means of DNB, in the increasing of information representation capacity, in the neural recruitment, in the synchronization of computations between neurons, in the neural modulation, in both scenarios, biological and artificial. These implications will also allow confirming the possible role of the NO on several neural circuits as the sleep-wake cycle control.

We will go onto these analysis about behavior of DNB, developing complementary studies as complex systems using bifurcation theory and analysis.

\section{REFERENCES}

Suárez Araujo, C.P, 2000. Study on the Functional and Organizational Role of the Neuromessenger Nitric Oxide in Learning: An Artificial and Biological Approach. In American Institute of Physics Press, Vol. 517, pp: 296-307

Suárez Araujo, C.P;, Fernández López P.; García Báez P. and Simoes da Fonseca J.L., 2006. A Model of Nitric Oxide Diffusion Based in Compartmental Systems. In International Journal of Computing Anticipatory Systems (IJCAS), Vol. 18, pp:172-186.

Hawkins, R.D., Kandel, E.R. and Siegelbaum, S.A., 1993. In Annu. ev. Neurosci. 16, 625-665.

Schuman, E.M. and Madison D.V., 1994. Nitric Oxide and Synaptic Function. In Annu. Rev. Neurosci. 17:153183

Zhuo, M., Laitinen, J.T., Li, X.C. And Hawkins, R.D., 1998. In Learn. Mem., 5, 467-480.

Garthwaite, J. and Boulton, C.L., 1995. Nitric Oxide Signalling in the Central Nervous System. In Аnnu. Rev. Physiol. 57 pp. 683-706.

Krekelberg, B., 1996. Nitric oxide in cortical map formation. In Journal of Chemical Neuroanatomy, 10, pp: 191-196.

Krekelberg, B., 1997. Modelling cortical seft-orgnization by volumen learning. Doctoral Dissertation. King's College London.

Krekelberg, B. and Taylor, J.G., 1998. A Dynamic Neighbourhood Function in Volume Learning. In Proceedings of the sixth annual conference on Computational neuroscience : trends in research, 1998: trends in research, 1998, pp 71-76.

Wood, J. and Garthwaite, J., 1994. Models of the Diffusional Spread of Nitric Oxide: Implications for Neural Nitric Oxide Signalling and its Pharmacological Properties. In Neuropharmacology Vol. 33, N. 11, pp. 1235-1244.

Anderson, D.H., 1983. Compartmental analysis and tracer kinetics. In Springer-Verlag, Berlin.

Syková, E., 2004. Extrasynaptic Volume Transmission and Diffusion Parameters of the Extracellular Space. In Neuroscience 129, pp 861-876.

Malinski, T. Taha Z., Grunfeld, S, Patton, S., Kapturczak, M. and Tombouliant, P., 1993. Difusion of nitric oxide in the aorta wall monitored in situ by porphyrinic microsensors. In Biochem Biophys Res Commun, 193 1076-1082 